\title{
Arabidopsis AtVPS15 is essential for pollen development and germination through modulating phosphatidylinositol 3-phosphate formation
}

\author{
Na Xu $\cdot$ Xin-Qi Gao $\cdot$ Xin Ying Zhao $\cdot$ \\ Dong Zi Zhu $\cdot$ Liang Zi Zhou $\cdot$ Xian Sheng Zhang
}

Received: 17 February 2011/ Accepted: 5 July 2011/Published online: 16 July 2011

(C) The Author(s) 2011. This article is published with open access at Springerlink.com

\begin{abstract}
Arabidopsis thaliana phosphatidylinositol 3kinase (AtVPS34) functions in the development and germination of pollen by catalyzing the biosynthesis of phosphatidylinositol 3-phosphate (PI3P). In yeast, Vps15p is required for the membrane targeting and activity of Vps34. The expression of Arabidopsis thaliana VPS15 (AtVPS15), an ortholog of yeast Vps15, is mainly detected in pollen grains and pollen tubes. To determine its role in pollen development and pollen tube growth, we attempted to isolate the T-DNA insertion mutants of AtVPS15; however, homozygous lines of atvps 15 were not obtained from the progeny of atvps15/+ heterozygotes. Genetic analysis revealed that the abnormal segregation is due to the failure of transmission of the atvps 15 allele through pollen. Most pollen grains from the atvps15/+ genotype are viable, with normal exine structure and nuclei, but some mature pollen grains are characterized with unusual large vacuoles that are not observed in pollen grains from the wild AtVPS15 genotype. The germination ratio of pollen from the atvps15/+ genotype is about half when compared to that from the wild AtVPS15 genotype. When supplied with PI3P, in vitro pollen germination of the atvps 15/+ genotype is greatly improved. Presumably,
\end{abstract}

$\mathrm{Na} \mathrm{Xu,} \mathrm{Xin-Qi} \mathrm{Gao,} \mathrm{and} \mathrm{Xin} \mathrm{Ying} \mathrm{Zhao} \mathrm{contributed} \mathrm{equally} \mathrm{to} \mathrm{this}$ paper.

Electronic supplementary material The online version of this article (doi:10.1007/s11103-011-9806-9) contains supplementary material, which is available to authorized users.

N. Xu · X.-Q. Gao $~ X$. Y. Zhao - D. Z. Zhu

L. Z. Zhou $\cdot$ X. S. Zhang $(\bowtie)$

State Key Laboratory of Crop Biology, Shandong Key

Laboratory of Crop Biology, College of Life Sciences, Shandong

Agricultural University, Taian 271018, Shandong, China

e-mail: zhangxs@sdau.edu.cn
AtVPS15 functions in pollen development and germination by regulating PI3P biosynthesis in Arabidopsis.

Keywords AtVPS15 - Pollen germination · Vacuole · Phosphatidylinositol 3-phosphate $\cdot$ Arabidopsis

\section{Introduction}

In higher plants, pollen grains hydrate, germinate, and protrude pollen tubes on the compatible stigma which then grow into the style towards the ovules. The two sperm cells move in the growing pollen tube to enter the female gametophyte for completion of the process of double fertilization. Failure of pollen germination or pollen tube formation will abort fertilization and further seed development. Signaling molecules and genes required for pollen germination and pollen tube growth have been identified in plants (Hepler et al. 2001; Johnson and Preuss 2002; McCormick 2004; Ge et al. 2010). Phospholipids, comprising about $1 \%$ of membrane lipids, play critical roles in regulating plant development and environment response (Xue et al. 2009). Phosphatidylinositol 3-phosphate (PI3P) is one type of phospholipid involved in receptor signaling and membrane/vesicle trafficking (Petiot et al. 2003; Roth 2004). In plant cells, PI3P is synthesized in the trans-Golgi/ endosome network and transported to the vacuole through the prevacuolar compartment (Kim et al. 2001). As an essential component of plant growth, PI3P signaling functions in diverse physiological processes such as pollen development, germination, and pollen tube growth (Lee et al. 2008a; Xue et al. 2009; Zhang and McCormick 2010).

Yeast Vps34 is a phosphatidylinositol 3-kinase (PI3K) catalyzing phosphoinositide to PI3P (Herman and Emr 
1990). Arabidopsis AtVPS34, a class III PI3K, is an ortholog of yeast Vps34 and is involved in the PI3P synthesis. Knockdown of AtVPS34 results in an embryo lethal phenotype in transgenic Arabidopsis (Welters et al. 1994). In root cells, the mutant of pi3K/AtVPS34 shows reduced endocytosis and reactive oxygen species production under salt stress (Leshem et al. 2007). Pollen grains of the T-DNA insertion mutants of AtVPS34 are sterile and are characterized by abnormal large vacuoles and a reduced number of nuclei (Lee et al. 2008b). In yeast, Vps34 forms a class III PI3K complex with Vps15p and Vps30 (ATG6) (Kihara et al. 2001). Yeast Vps15p is a Ser/Thr protein kinase containing HEAT repeats within its central region and a series of WD-40 domains within its C-terminus (Stack et al. 1993). Vps15p is required for the membrane targeting and activity of Vps34 (Yan and Backer 2007). The Arabidopsis AtVPS15 (At4g29380) was identified as the putative ortholog of yeast Vps15 (Mueller-Roeber and Pical 2002); however, its function in Arabidopsis development is unknown. In this study, we found that AtVPS15 was mainly detected in anthers. The pollen of the AtVPS15 mutants is defective in vacuolar morphology and pollen germination, but an exogenous supply of PI3P could restore pollen germination of mutants. Apparently, AtVPS15 regulates the formation of PI3P and plays an essential role during the development and germination of pollen grains.

\section{Results}

AtVPS15 appears to be an ortholog of yeast Vps15p and human $\mathrm{hVps} 15$

Arabidopsis AtVPS15 (At4g29380) contains 11 exons (Fig. 1a). The AtVPS15 predicted protein shares 22 and $27 \%$ identity overall with Vps15p of Saccharomyces cerevisiae and $\mathrm{hVps} 15$ of Homo sapiens, respectively (Herman et al. 1991a; Panaretou et al. 1997). The percent identity is much greater within conserved regions throughout the protein (Online Resource 1). Similar to Vps15p and hVps15, the N-terminus of AtVPS15 also contains the putative catalytic PKc domain of the protein kinase C (Fig. 1b). The lysine (K54, K149) and aspartic acid (D147) residues in the N-terminus of Vps15p are important for its enzymatic activity and vacuole localization (Herman et al. 1991b). These residues are strictly conserved in human hVps15. Similarly, the AtVPS15 protein is conserved also for these amino acid residues (K54, D149, and K151) (Online Resource 1). The N-terminus of Vps15p, namely (Metl)-Gly2-Ala3-Gln4-Leu5Ser6-Leu7, could be myristoylated, where the glycine residue is required for this process (Herman et al. 1991a, b). This myristoylation site is also conserved in AtVPS15 (Online Resource 1). In addition, Vps15p contains HEAT repeats, which interact with $\mathrm{Vps} 34$, at the central region, and a series of C-terminus WD-40 domains. There are two HEAT repeats and three WD-40 domains at the central and $\mathrm{C}$ terminus of the AtVPS15 protein (Fig. 1b and Online Resource 1). AtVPS15 protein is highly conserved in eukaryotes, including higher plants such as Populus trichocarpa, and Vitis vinifera, as well as with some lower plants (Fig. 1c).

Transcription of AtVPS15 and subcellular localization of AtVPS15

The qRT-PCR was employed to determine the expression pattern of AtVPS15 in Arabidopsis. AtVPS15 was abundantly expressed in anther tissues but low in other tissues and organs (Fig. 2a). We further investigated the AtVPS15 expression in anthers, pollen grains, and pollen tubes using in situ hybridization. The AtVPS15 cDNA was barely detected in microsporocytes of anthers at stage 6; however, its accumulation was high in tetrads at stage 7 , microspores, mature pollen grains, and tapetum cells (Fig. 2b). AtVPS15 transcript also was detectable in the growing pollen tubes on the stigma (Fig. 2b). These results indicate that AtVPS15 may function in pollen development and pollen tube growth. The YFP-AtVPS15 fusion protein was used to localize the AtVPS15 protein in the tobacco pollen tubes. The fluorescent signal of YFP-AtVPS15 (from Lat52::YFP-AtVPS15) was distributed uniformly in the cytoplasm, which was similar to that of the empty-vector control (Lat52::YFP) (Fig. 2c).

The atvps 15 mutation reduces male gametophyte transmission

To study the function of AtVPS15 in Arabidopsis development, we used two T-DNA insertion lines of AtVPS15, GABI_015F04 (insertion in the 8th exon) and SALK_004719 (insertion in the 4th intron). The T-DNA insertions were confirmed by PCR analysis using the AtVPS15-specific primers and T-DNA-specific primers (Fig. 1a). Only plants heterozygous for the AtVPS15 mutation were identified after screening 40 and 35 seedlings of GABI_015F04 and SALK_004719, respectively. The heterozygous lines of GABI_015F04 and SALK 004719 were designated as atvps15-1/+ and atvps15-2/+, respectively. Segregation analysis of the selfed progeny revealed that the ratios of wild-type/heterozygous plants from the atvps15-1/+ and atvps 15-2/+ genotypes were 1.16:1 and 1.22:1, respectively (Table 1). No homozygous progeny were found for the AtVPS15 mutations. Therefore, the two independent T-DNA insertions in AtVPS15 lead to 
(A)

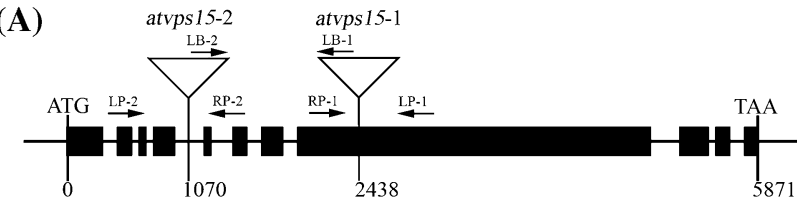

(B)

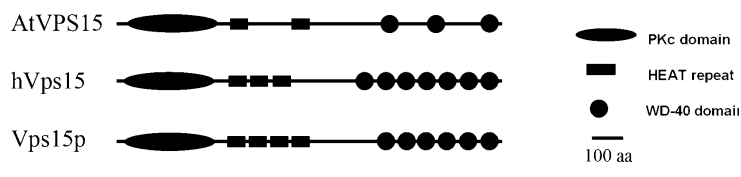

(C)

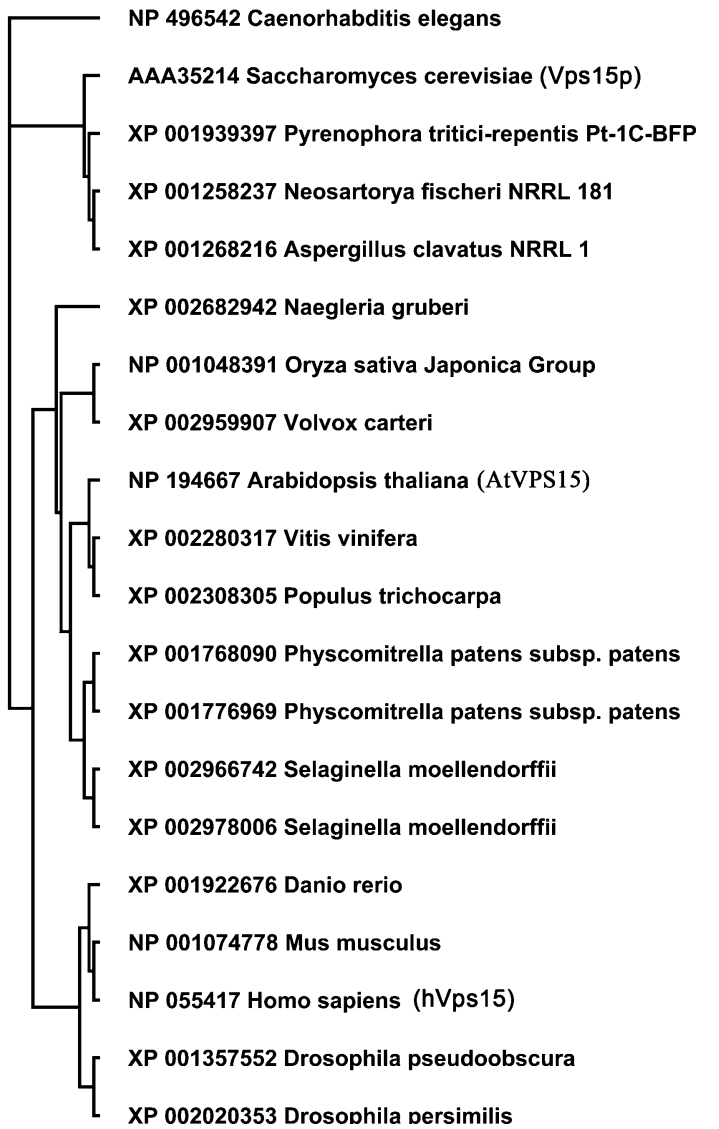

Fig. 1 Molecular characterization of AtVPS15 and identification of atvps15-1/+ and atvps15-2/+ insertion lines. a Schematic representation of AtVPS15 and atvps15-1/+ and atvps15-2/+. Exons of AtVPS15 are represented by black boxes and introns by lines. The locations of the T-DNA insertion in the mutant lines are indicated with triangles. LBs primers for left border of the T-DNA, RPs and $L P s$ gene-specific primers at downstream and upstream locations, respectively. b Predicated domains of the AtVPS15, yeast Vps15p (Saccharomyces cerevisiae, Genbank No. AAA35214), and human hVps15 (Homo sapiens, Genbank No. NP055417) proteins. c Phylogenetic analysis of AtVPS15 with its orthologs of eukaryotic proteins

segregation distortion indicating a gametophytic defect. Reciprocal crosses between wild-type plants and heterozygous mutants were carried out to test gametophytic transmission efficiency. About $88.0 \%$ and $89.2 \%$ of the mutant allele was transmitted via the female gametophytes of atvps15-1/+ and atvps15-2/+, respectively, but no

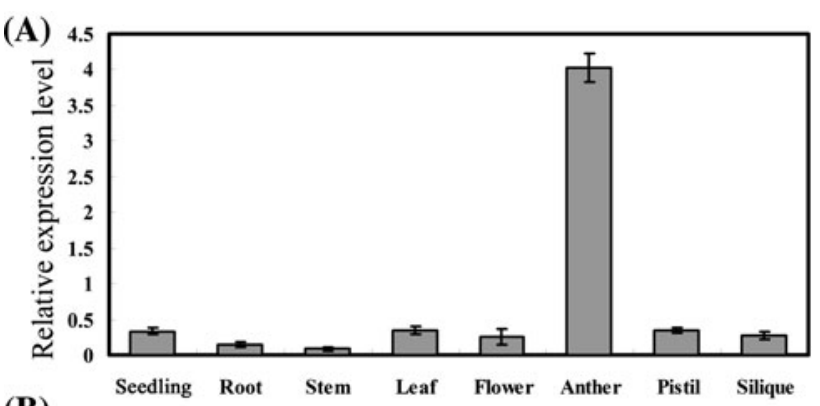

(B)
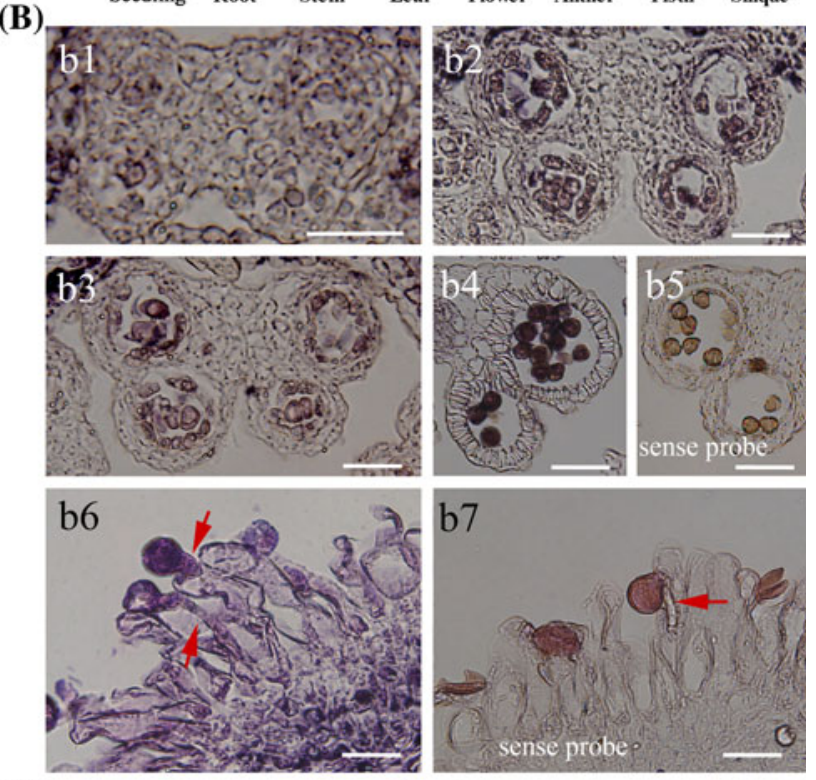

(C)

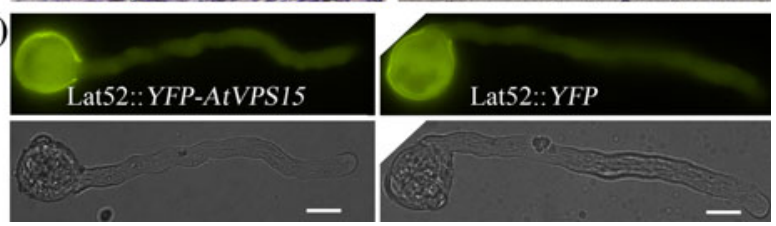

Fig. 2 Expression pattern of AtVPS15 and subcellular localization of AtVPS15. a Analysis of the relative expression level of AtVPS15 in different organs of Arabidopsis by qRT-PCR. The experiment was repeated three times; error bars represent the standard errors of the means. b RNA in situ hybridization analysis for AtVPS15 transcription in microsporocytes of anthers at stage $6(b 1)$, tetrads at stage 7 $(b 2)$, the microspores (b3), the tapetum cells ( $b 2$ and $b 3)$, the mature pollen grains ( $b 4$ ), and the pollen tubes ( $b 6$ ) of Arabidopsis. $b 5$ and $b 7$ are the sense probe controls. Arrows indicate the pollen tubes on the surface of stigma. c Transient transfection of Lat52::YFP-AtVPS15 and Lat52::YFP constructs in tobacco pollen tube. Bars $50 \mu \mathrm{m}$ in b1$b 5,20 \mu \mathrm{m}$ in $b 6$ and $b 7$, and $10 \mu \mathrm{m}$ in $\mathbf{c}$

transmission was observed via the male gametophytes (Table 2). Clearly, AtVPS15 is necessary for the development of the fertile male gametophyte.

Pollen grains carrying the atvps 15 mutation are defective for pollen germination

The mature pollen viability was examined for heterozygous mutants of atvps15-1/+ and atvps15-2/+ using the 
Table 1 Segregation analysis of selfed progeny of atvps15/+ mutants

\begin{tabular}{lllll}
\hline Genotype $^{\mathrm{a}}$ & AtVPS15/+ & atvps15/+ & atvps15/- & Ratio \\
\hline $\begin{array}{c}\text { atvps15-1/+ } \\
\text { self-fertilized }\end{array}$ & 158 & 138 & 0 & $1.16: 1: 0$ \\
$\begin{array}{c}\text { atvps15-2/+ } \\
\text { self-fertilized }\end{array}$ & 70 & 57 & 0 & $1.22: 1: 0$ \\
\hline
\end{tabular}

a The genotype of selfed progeny of atvps $15 /+$ was analyzed by a PCR-based method as described in "Materials and Methods"

Table 2 Analysis of the genetic transmission of atvps 15 alleles

\begin{tabular}{lccc}
\hline Genotype (Female $\times$ Male) & atvps15/+ & AtVPS15/+ & TE $(\%)$ \\
\hline atvps15-1/ $\times$ Col-0 & 73 & 83 & $88.0^{\mathrm{a}}$ \\
WT $\times$ atvps15-1/+ & 0 & 101 & 0 \\
atvps15-2/ $+\times$ WT & 58 & 65 & 89.2 \\
WT $\times$ atvps15-2/+ & 0 & 189 & 0 \\
\hline
\end{tabular}

a The genetic transmission of atvps 15 was analyzed by genotyping F1 progeny of the denoted crosses. The transmission efficient $(\mathrm{TE})=($ atvps $15 /+) /($ AtVPS15/+ $) \times 100 \%$ was calculated according to Howden et al. (1998)

Alexander's staining procedure (Alexander 1969). All mature pollen grains of atvps15/+ heterozygous mutants were red after staining (Fig. 3a), indicating that they were still viable up to this stage. In addition, the propidium iodide dyeing and scanning electron microscopy analysis revealed that the pollen exines of atvps 15-1/+ and atvps 15-2/+ lines were normal as that of the wild-type plants (Online Resource 2). Therefore, we decided to check whether pollen nuclear division is defective using DAPI labeling. Most pollen grains had three nuclei, and there was no significant difference in numbers of nuclei among the wild-type and heterozygous mutants (Fig. 3b, c). Taken together, the atvps 15 mutation did not cause detectable abnormality of the pollen grains. We further examined the germination of mature pollen from wild-type plants, atvps 15-1/+ line, and atvps $15-2 /+$ line. About $90.3 \%$ of the pollen grains from wild type could germinate after $4 \mathrm{~h}$ on pollen germination medium. In contrast, the germination ratios were $44.5 \%$ and $44.7 \%$ of atvps $15-1 /+$ and atvps $15-2 /+$ lines, respectively (Fig. 3d, e). These data implied that pollen grains carrying the atvps 15 mutation could not germinate and were responsible for the reduced male transmission.

The atvps 15 mutation affects vacuolar organization of pollen

The AtVPS34 (PI3K) protein is important for vacuolar organization, and the absence of AtVPS34 results in large vacuoles in pollen after the first mitosis (Lee et al. 2008b). Since Vps34 requires the Vps15p to be fully functional, it was of interest to investigate the vacuolar organization in pollen grains of the atvps 15 mutants. We sectioned anthers of different developmental stages in wild-type and atvps 15/ + lines. At the early stage of bicellular pollen, one or several large vacuoles were observed in the wild-type, atvps15-1/+ and atvps15-2/+ mutants, and no structural difference was discovered in all tested lines (Fig. $4 a-c)$. In the late stage of bicellular pollen, the large vacuoles disappeared, and many small ones were seen in wild-type pollen grains (Fig. 4d); however, the large vacuoles remained in some pollen grains in atvps15/+ plants (Fig. 4e, f). At the mature tricellular stage of pollen grains, the vacuoles in wild-type pollen became smaller than that at the bicellular stage (Fig. 4g), while large vacuoles with irregular morphology were still present in some pollen grains in the atvps $15 /+$ anthers (Fig. 4h, i). In comparison, all pollen grains had normal vacuoles in the examined wild-type anthers at the mature tricellular stage, but $13.8 \%$ and $22.4 \%$ of pollen grains displayed irregular vacuoles in the atvps15-1/+ and atvps15-2/+ mutants, respectively (Table 3). Transmission electron microscopy revealed that, in mature pollen grains, the large and irregular vacuoles were present in the mutant plants but not in the wild-type plants (Fig. 4j-o). The large vacuoles contained deposits in their lumen that might be degraded cytoplasm (Fig. 4n, o; Lee et al. 2008; Whitley et al. 2009).

Exogenous PI3P supply restores pollen germination of the atvps 15 mutants

Both atvps 15 and atvps 34 mutants displayed similar phenotypes in vacuolar organization and pollen germination indicating that they are in a common pathway. As reported for yeast (Stack et al. 1993), Vps34 converts the phosphoinositide to PI3P, and Vps15p regulates the Vps34 activity. We hypothesized that the knockout of AtVPS15 impairs ATVPS34 activity and interferes with PI3P synthesis in pollen, which prevents pollen germination. Therefore, the germination of pollen from the atvps15/+ lines was examined on normal germination media supplemented with PI3P and its carrier (Echelon, USA) and normal germination media supplemented with carrier only. The PIP3 and its carrier form complex that facilitates the transportation of PI3P into cells. The pollen germination of the wild-type and atvps 15/+ lines did not show significant change on medium with carrier only (Figs. 5a, 3e). About 79.4 and $78.9 \%$ of pollen grains from atvps $15-1 /+$ and atvps 15-2/+ lines, respectively, germinated on the PI3Psupplemented media, whereas the germination ratio of wild-type pollen was reduced under the same conditions (Fig. 5b, c). Therefore, the exogenous PI3P supply restores the pollen germination in atvps 15 mutants, and the mutation of AtVPS15 reduces the PI3P level in pollen that prevents the pollen germination in the atvps 15 mutants. 
Fig. 3 Pollen viability and germination of atvps15/+ lines and wild-type (WT) plants. a Alexander's staining to detect the viability of mature pollen. b Mature pollen grains stained with DAPI showing that most pollen contained three nuclei. Bars $10 \mu \mathrm{m}$. c Statistics of the mature pollen with three nuclei. The experiment was repeated three times; error bars represent the SE of the mean. $\mathrm{n}=325$ (wild-type), 364 (atvps15-1/+), and 346 (atvps 15-2/+). d Pollen germination on medium.

e Statistics of the germination ratio of the pollen on medium. The experiment was repeated three times; error bars represent the standard error of the mean; $\mathrm{n}=1,636$ (wild-type), 1,660 (atvps15-1/+), and 1353 (atvps15-2/+)
(A)
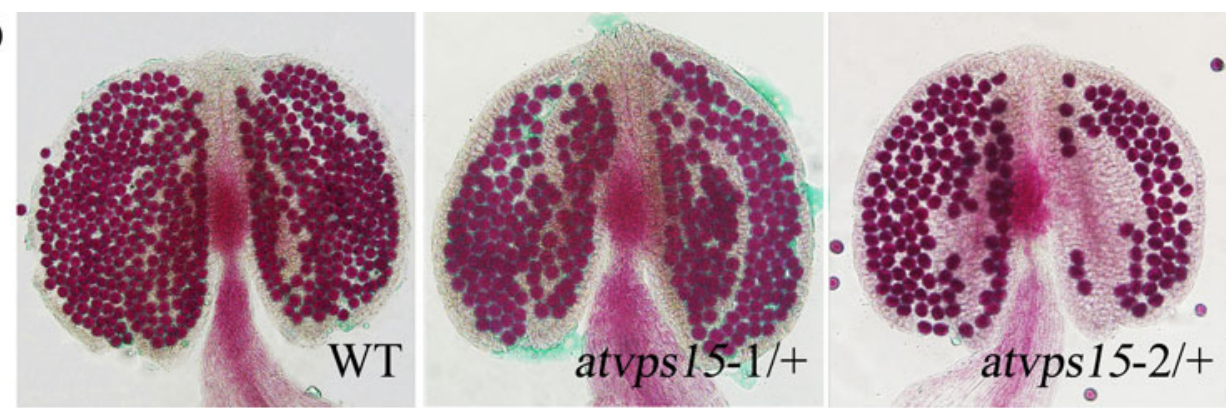

(B)
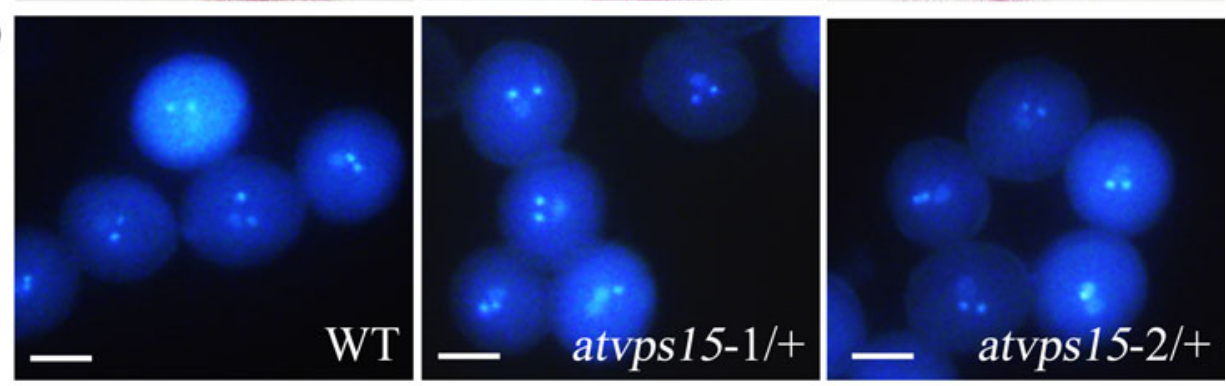

(C)

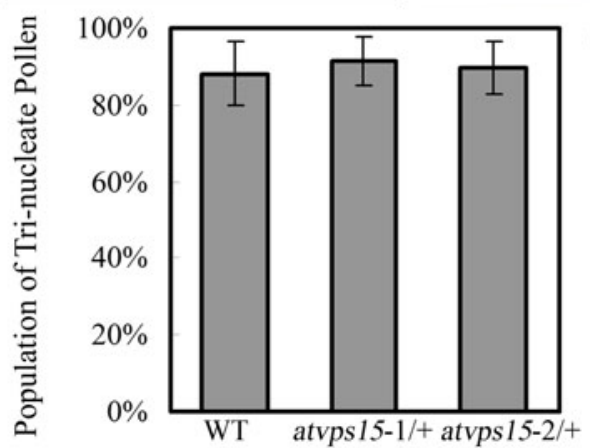

(E) $100 \%$

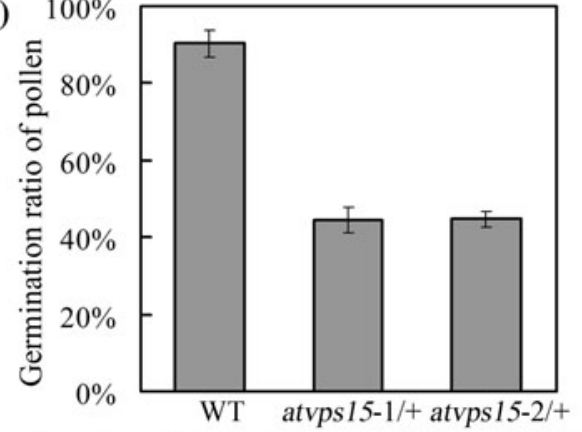

(D)
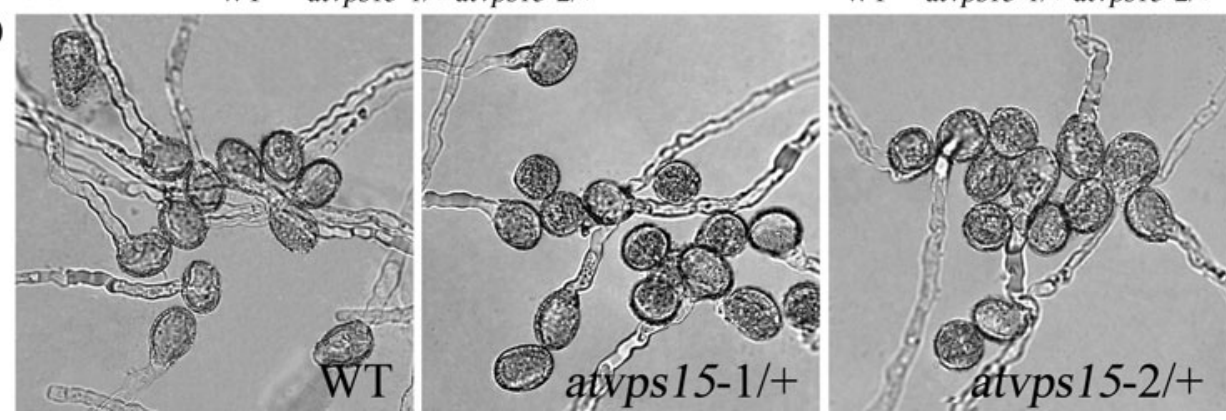

\section{Discussion}

Phosphoinositides function in membrane trafficking and polarized growth of pollen tube (Monteiro et al. 2005; Samaj et al. 2006; Zonia and Munnik 2008; Ischebeck et al. 2008; Sousa et al. 2008). In yeast, Vps15p plays its role by forming a class III PI3K complex with Vps34 and Vps30. Vps34 has lipid kinase activity and functions in PI3P synthesis (Herman and Emr 1990). In Arabidopsis, the atvps 34 mutation impairs the production of PI3P and results in failure of pollen germination (Lee et al. 2008b). In yeast, Vps15 is required for membrane targeting and function of Vps34 (Stack et al. 1993). Our results revealed that AtVPS15 is important for pollen development and germination, and further that an exogenous supply of PI3P improves the pollen germination of the atvps 15 mutants. Due to the close phenotype between the atvps 15 mutants and the atvps34 mutants, AtVPS15 potentially interacts with AtVPS34 and regulates AtVPS34 activity and PI3P synthesis in vivo, as in yeast (Stack et al. 1993). Additionally, AtAtg6 (AtVPS30) plays roles in pollen germination of Arabidopsis. The mutation of AtAtg6 leads to failure of pollen germination (Fujiki et al. 2007; Qin et al. 2007; Harrison-Lowe and Olsen 2008). PI3K complex is involved in autophagy and vacuolar protein sorting (VPS) in yeast (Yorimitsu and Klionsky 2005). However, whether 
Fig. 4 Vacuolarization in bicellular and tricellular pollen grains of atvps $15 /+$ lines and wild-type (WT) plants. Pollen grains at the early bicellular (a through c), late bicellular (d through f) and mature (g through o) stages of wildtype (left lane), atvps15-1/+ (middle lane), and atvps15-2/+ (right lane) plants. $\mathbf{a}-\mathbf{i}$ are the photos of semi-thick sections; $\mathbf{j}-\mathbf{0}$ are the photos of ultrathin sections by transmission electron microscopy. $\mathbf{m}-\mathbf{0}$ are the higher magnifications of the square areas in $\mathbf{j}-\mathbf{l}$, respectively. Arrowheads indicate the pollen grains containing abnormal vacuoles of atvps $15 /+$ lines. Arrows indicate the deposits in irregular vacuoles of atvps15/+ pollen grains. $v$ vacuole. Bars $20 \mu \mathrm{m}(\mathbf{a}-\mathbf{i}), 2 \mu \mathrm{m}(\mathbf{j}-\mathbf{l})$, and $1 \mu \mathrm{m}(\mathbf{m}-\mathbf{o})$
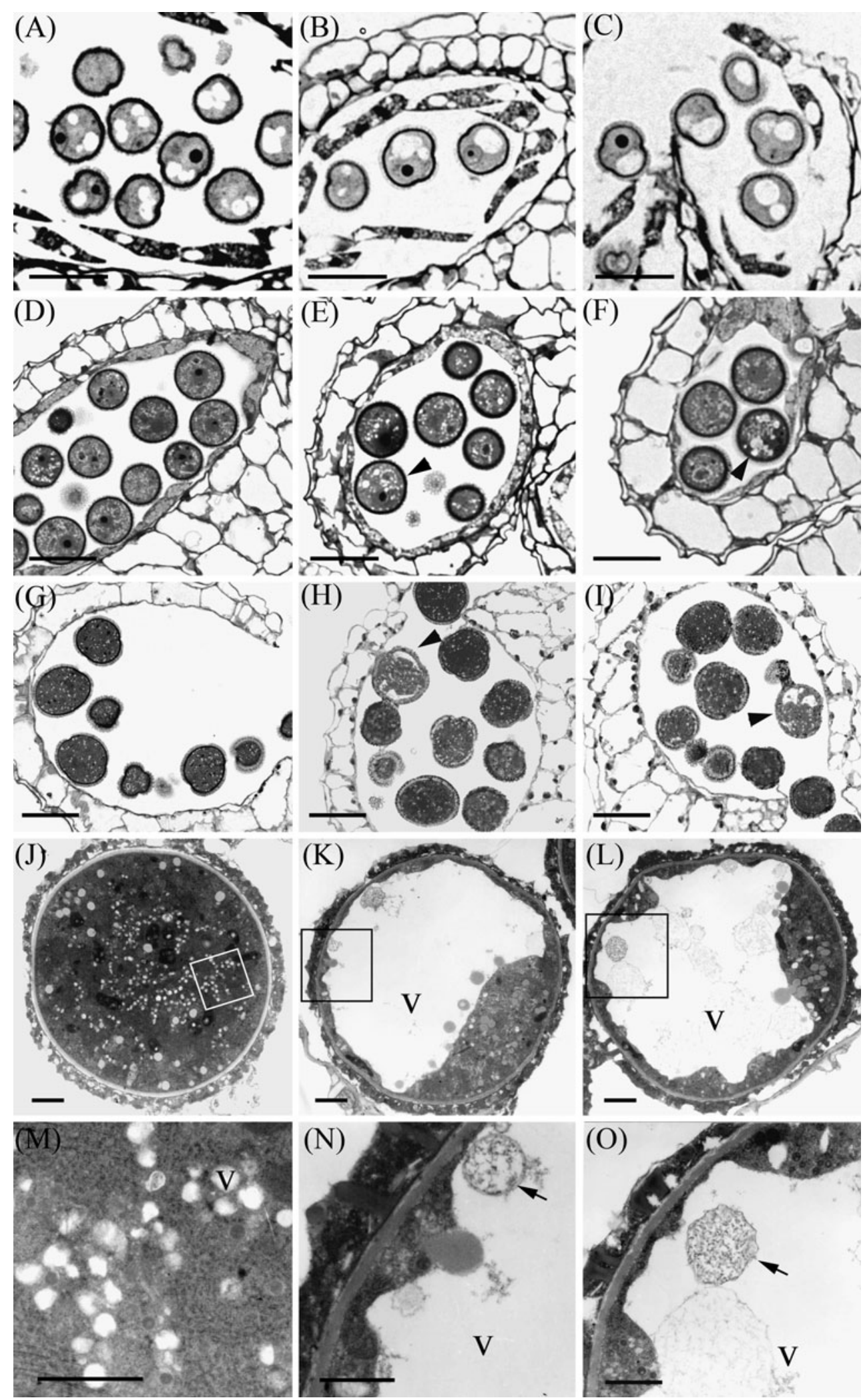

the Arabidopsis Atg-regulated autophagy plays a role in pollen germination remains unknown (Fujiki et al. 2007; Qin et al. 2007; Harrison-Lowe and Olsen 2008).
In Arabidopsis, PI3K can phosphorylate the phosphatidylinositol at the D-3 position to generate the PI3P, and then the PIKfyve/Fab1 family will phosphorylate the PI3P 
Table 3 Population of mature pollen with abnormal vacuoles of atvps $15 /+$

\begin{tabular}{lc}
\hline Genotype & Abnormal pollen \\
\hline WT & $0 \%(0 / 68)$ \\
Atvps15-1/+ & $13.84 \pm 0.48 \%(15 / 123)^{*}$ \\
Atvps15-2/+ & $22.41 \pm 2.51 \%(17 / 94)^{*}$ \\
\hline
\end{tabular}

The mature pollen grains with abnormal vacuoles were observed and counted from the semi-thin sections as described in "Materials and Methods". Actual numbers of pollen grains counted are indicated in parentheses (Abnormal pollen/total count). The results from three independent experiments were combined for statistical analysis (Student's t test, $* P<0.01$ )

at the D-5 position to produce the phosphatidylinositol 3,5bisphosphate $\left(\mathrm{PI}(3,5) \mathrm{P}_{2}\right)$ (Cantley 2002). Arabidopsis FAB1A and FAB1B are orthologs of the PIKfyve/Fab1 family proteins. The Arabidopsis fabla/fablb mutants are defective in pollen development and germination, which may be caused by the decrease of the $\mathrm{PI}(3,5) \mathrm{P}_{2}$ level in pollen (Whitley et al. 2009). ATVPS15 may affect the $\mathrm{PI}(3,5) \mathrm{P}_{2}$ synthesis in pollen by regulating ATVPS34 activity and the PI3P level. The similar vacuolar phenotype of atvps 15, atvps34 and fabla/fablb mutants provides an additional line of evidence. Studies in non-plant cells revealed that $\mathrm{PI}(3,5) \mathrm{P}_{2}$ mediates the fission of vacuoles (or its equivalent organelles) by increasing the surface-tovolume ratio of intracellular organelles (Weisman 2003; Sbrissa and Shisheva 2005). Although the atvps15, atvps 34 , and fabla/fablb mutants are similar in pollen germination, they differ in other stages during the pollen development. The atvps 34 and fabla/fablb mutants associate with reduced pollen viability, but some pollen grains of fabla/fablb mutant are collapsed; however, atvps 15 pollen had normal viability and morphology. Additionally, the atvps 15 mutants associate with the largest fraction of pollen with normal vacuoles, while the fabla/fablb mutant has the least fraction of pollen with normal vacuoles. The different levels of $\mathrm{PI}(3,5) \mathrm{P}_{2}$ in these mutants may lead to
Fig. 5 Pollen germination of atvps 15/+ heterozygous and wild-type (WT) plants on media supplemented with exogenous PI3P carrier with and without PI3P. a Pollen germination on the medium adding exogenous carrier for PI3P. b Pollen germination on the medium adding exogenous PI3P and its carrier. c Statistics of pollen germination on the medium containing PI3P and its carrier. The experiment was repeated three times; error bars represent the standard error of the mean. $\mathrm{n}=816$ (wild-type), 800 (atvps 15-1/+), and 837 (atvps15-2/+) on medium containing carrier and $=810$ (wild-type), 874 (atvps15-1/+), and 842 (atvps15-2/+) on medium containing PI3P and carrier
(A)
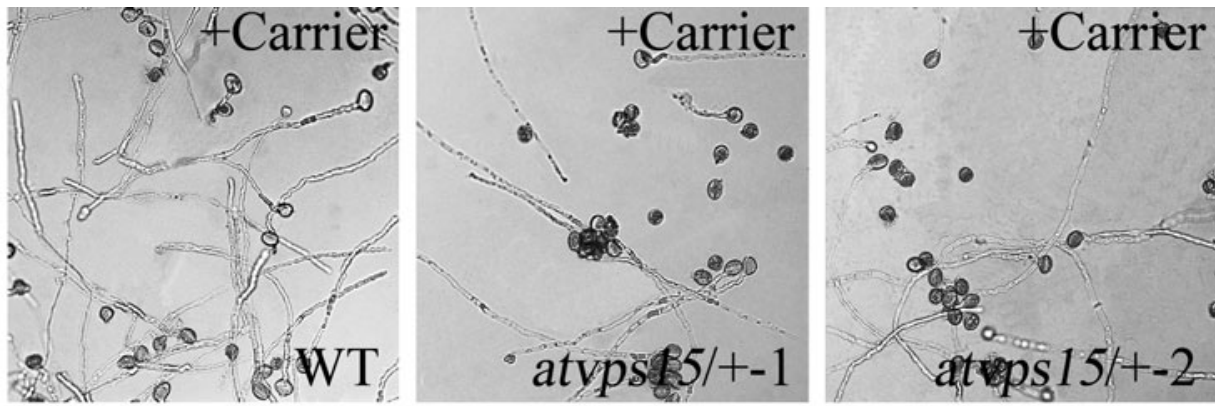

(B)
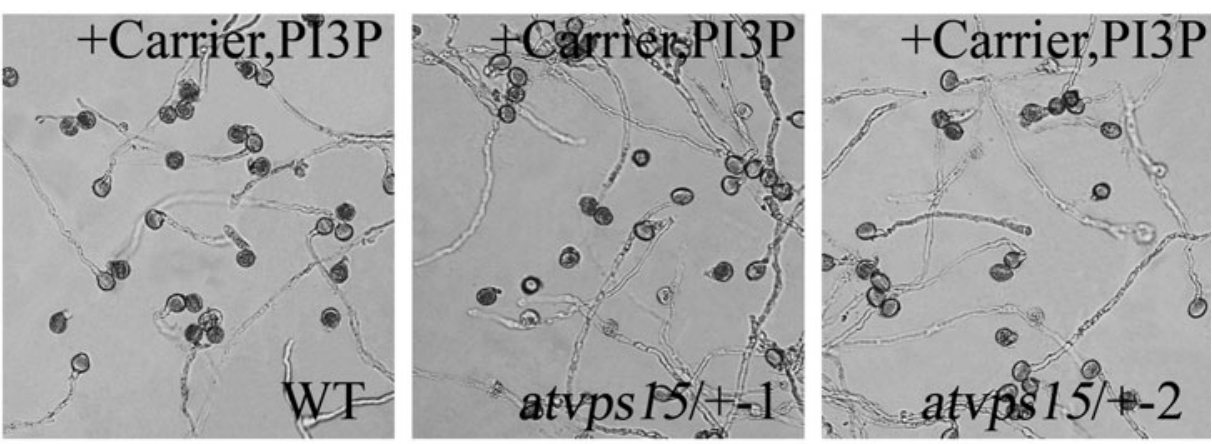

(C)

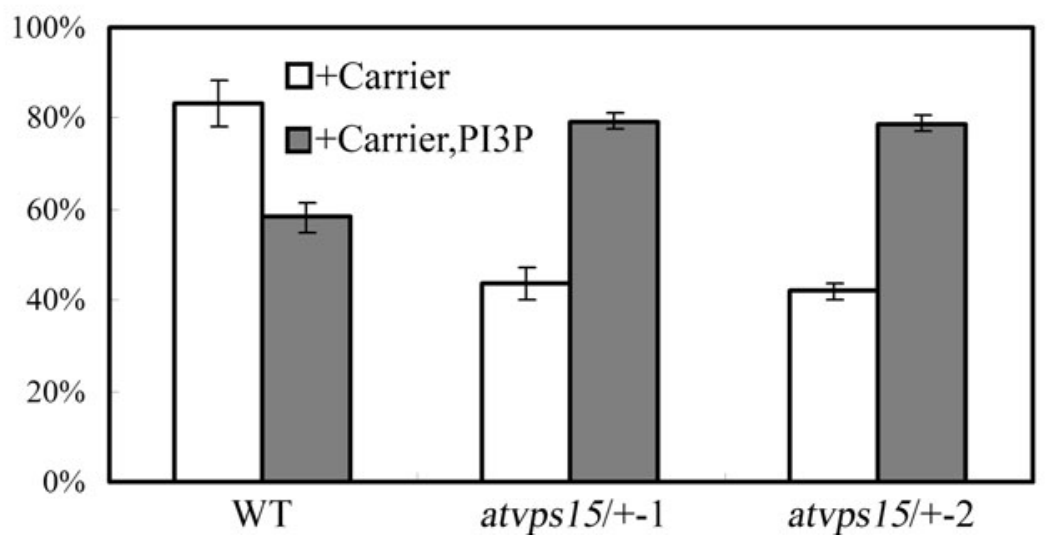


different phenotypes in pollen development. How ATVPS15, ATVPS34, and FAB1A/FAB1B contribute to the $\mathrm{PI}(3,5) \mathrm{P}_{2}$ accumulation in pollen is likely conditioned by their position in the $\mathrm{PI}(3,5) \mathrm{P}_{2}$ metabolism pathway. FAB1A/FAB1B directly catalyzes the production of $\mathrm{PI}(3,5) \mathrm{P}_{2}$ and the fabla/fablb mutations cause significant changes in $\mathrm{PI}(3,5) \mathrm{P}_{2}$ level. ATVPS15 may only cause minor change in $\mathrm{PI}(3,5) \mathrm{P}_{2}$ level since the ATVPS15 controls the PI3P accumulation by regulating the ATVPS34 activity. Alternatively, AtVPS15, AtVPS34, and FAB1A/ FAB1B may be involved in other pathways that regulate pollen development and germination.

\section{Materials and methods}

Plant materials

Two T-DNA insertion lines of Arabidopsis thaliana (Col-0 ecotype) of AtVPS15 (GABI_015F04 and SALK_004719) were obtained from NASC and ABRC, respectively. The genotypes of T-DNA insertion line plants were determined by a PCR-based method. The following primers were used: LB1 (5'-ATATTGACCATCATACTCATTG-3'), LP1 (5'CTCCGTCTGATCACTCAAAGC - $3^{\prime}$ ), and RP1 (5'-CA ATGAACGCAAGCACAGTAC-3') for GABI_015F04 and LB2 (5'-ATTTTGCCGATTTCGGAAC-3'), LP2 (5'GAATGATTTTGCAGCTTCTGG-3'), and RP2 (5'-CA AGAGAATCGATGCGAAAAG-3') for SALK_004719. The wild-type Arabidopsis and T-DNA insertion plants were grown at $22^{\circ} \mathrm{C}$ in a $16 / 8 \mathrm{~h}$ light/dark cycle.

Pollen analysis and microscopy

For pollen staining, mature pollen grains or the anthers were used. The mature anthers were soaked in Alexander stain for 1-2 days to detect pollen viability according to Alexander (1969) under a BX51 microscope (Olympus, www.olympus.com). To observe the exines of pollen grains, we used $10 \mu \mathrm{g} / \mathrm{ml}$ (w/v) propidium iodide (Sigma, www.sigmaaldrich.com) to stain mature pollen grains followed by observation under Zeiss CLSM 510 meta (www.zeiss.com), according to Tian et al. (2006). The nuclei of pollen grains were stained with $1 \mu \mathrm{g} / \mathrm{ml}(\mathrm{w} / \mathrm{v})$ DAPI (Sigma, www.sigmaaldrich.com) for $30 \mathrm{~min}$ and observed by epifluorescence microscopy (Olympus BX51 microscope). For the sections of anthers and pollen grains, the materials were embedded in Spurr resin and after-fixed with a solution of $4 \%(\mathrm{w} / \mathrm{v})$ glutaraldehyde and $1 \%(\mathrm{w} / \mathrm{v})$ $\mathrm{OsO}_{4}$ in phosphate buffer (pH 7.2). The semi-thick sections were stained with $0.5 \%(\mathrm{w} / \mathrm{v})$ toluidine blue-O (Sigma, www.sigmaaldrich.com) and observed with an Olympus BX51 microscope.
Pollen germination in vitro

To analyze pollen germination in vitro, the pollen grains at anthesis of wild-type Arabidopsis and T-DNA insertion lines were collected and cultured on medium containing $1 \mathrm{mM} \mathrm{CaCl}_{2}, 1 \mathrm{mM} \mathrm{Ca}\left(\mathrm{NO}_{3}\right)_{2}, 1 \mathrm{mM} \mathrm{MgSO} \mathrm{m}_{4}, 0.01 \%$ (w/v) $\mathrm{H}_{3} \mathrm{BO}_{3}$, and $18 \%(\mathrm{w} / \mathrm{v})$ sucrose solidified with $0.5 \%(\mathrm{w} / \mathrm{v})$ agar ( $\mathrm{pH} 7.0)$. To analyze the role of PIP3 in pollen germination, PIP3 (PtdIns(3)P di- $\left.\mathrm{C}_{16}, \mathrm{P}-3016\right)$ and its carrier (Carrier 3, P-9C3) (Echelon Biosciences Inc, www.echeloninc.com) were added into the pollen germination medium according to the manufacturer's recommendation. In medium, the ratio of PIP3 and carrier is 1:1 to form the PIP3carrier complex at a final concentration of $6.25 \times 10^{-3} \mathrm{mg} /$ $\mathrm{ml}$. The pollen grains were cultured immediately at $28^{\circ} \mathrm{C}$, $100 \%$ relative humidity, and cool light at $30 \mathrm{mmol} / \mathrm{m}^{2} \mathrm{~s}$. The germinated pollen grains were counted and images were captured at $4 \mathrm{~h}$ after germination.

Quantitative Real-time PCR (qRT-PCR)

The Arabidopsis seedlings of 3-week-old (for seedling, root and leaf) and 5-week-old (for stem, flower, anther, pistil and silique) were collected for AtVPS15 expression analysis. Total RNA was isolated with the Trizol isolation reagent (Invitrogen, www.invitrogen.com) for reverse transcription. qRT-PCR was conducted to determine AtVPS15 transcript levels with the primer pairs VPS15RT-sense (5'-GGAA GTTACCAAGCAATAAGG-3') and VPS15RT-antisense (5'- AGAGCGGATACCAGGAAG -3'). Tubulin was used as a quantitative control and amplified using the primers, TUB2-RT-sense (5'-ATCCGTGAAGAGTACCCAGAT-3') and TUB2-RT-antisense (5'-AAGAACCATGCACTCAT CAGC $\left.-3^{\prime}\right)$. The line arrangement of the PCR products was established by comparing samples that were run for different numbers of cycles. All experiments were repeated three times.

Transient expression YFP fusion protein in tobacco pollen tubes

Transient expression of YFP-AtVPS15 fusion protein in pollen tubes of tobacco was used to study the intracellular localization of AtVPS15. The full-length cDNA of AtVPS15 was amplified by PCR using GATEWAY-compatible primers VPS15-sense (5'-CACCATGGGAAACA AAATCGCTCGTACGACAC-3') and VPS15-antisense (5'-TTACTTCCAGACCTTTATGGCTCCATCT-3') and cloned into the pENTY/D-TOPO vector (Gateway, Invitrogen, www.invitrogen.com) for sequencing, and then recombined into pAL20 to create Lat52::YFP-AtVPS15 construct. The Lat52::YFP construct was used as the control. Each construct was introduced into tobacco (Nicotiana 
tabacum) pollen using a pneumatic particle gun (PDS1000/He; Bio-Rad, www.bio-rad.com), as described by Zhang and McCormick (2007). After 4-8 h cultivation on medium, the pollen grains were visualized under a fluorescence microscope (Olympus BX51).

RNA in situ hybridization

RNA in situ hybridization was performed as previously described by Li et al. (2010). The specific AtVPS15 probe is a 570-bp fragment amplified in the coding region of AtVPS15 with the primers VPS15-IF (5'-TCACAAGGGA GGTAGTTTATCG-3') and VPS15-IR (5'-GCTTAGGCA CTGGGACCGAACT- $3^{\prime}$ ). The samples were observed using an Olympus BX51 microscope.

\section{Electron microscopy}

For the observation of pollen morphology, mature pollen grains coated with gold palladium were observed under a JSM-6610LV scanning electron microscope (JEOL, Japan) and photographed. Transmission electron microscopy was performed as described by Wang et al. (2009), with modification. The mature anthers were fixed in $4 \%(\mathrm{v} / \mathrm{v})$ glutaraldehyde and $1 \%(\mathrm{w} / \mathrm{v})$ osmic acid (Sigma). After washing, the specimens were dehydrated in a graded ethanol series and embedded in Epon 812 resin (SPI-CHEM, USA). Ultrathin sections were stained with acetate uranium and lead citrate. The specimens were observed using a JEM1200EX transmission electron microscope (JEOL, Japan).

Acknowledgments We thank Dr. Yan Zhang (Shandong Agricultural University) for her helpful discussions and providing pAL20 vector, and Dr. Sharman D. O'Neill (University of California, Davis, USA) for her critical reading for the manuscript. We thank Mr. YanKui Guo (Shandong Agricultural University) for his kind help with electron microscopy. We are also grateful to the Arabidopsis Biological Resource Center and the European Arabidopsis Stock Centre for kindly providing the Arabidopsis mutant seeds. This study is funded by the Major Research Plan from the Ministry of Science and Technology of China (No. 2007CB947600).

Open Access This article is distributed under the terms of the Creative Commons Attribution Noncommercial License which permits any noncommercial use, distribution, and reproduction in any medium, provided the original author(s) and source are credited.

\section{References}

Alexander MP (1969) Differential staining of aborted and nonaborted pollen. Stain Technol 44:117-122

Cantley LC (2002) The phosphoinositide 3-kinase pathway. Science 296:1655-1657

Fujiki Y, Yoshimoto K, Ohsumi Y (2007) An Arabidopsis homolog of yeast ATG6/VPS30 is essential for pollen germination. Plant Physiol 143:1132-1139
Ge X, Chang F, Ma H (2010) Signaling and transcriptional control of reproductive development in Arabidopsis. Curr Biol 20:988-997

Harrison-Lowe NJ, Olsen LJ (2008) Autophagy Protein 6 (ATG6) is required for pollen germination in Arabidopsis thaliana. Autophagy 4:339-348

Hepler PK, Vidali L, Cheung AY (2001) Polarized cell growth in higher plants. Annu Rev Cell Dev Biol 17:159-187

Herman PK, Emr SD (1990) Characterization of VPS34, a gene required for vacuolar protein sorting and vacuole segregation in Saccharomyces cerevisiae. Mol Cell Biol 10:6742-6754

Herman PK, Stack JH, DeModena JA, Emr SD (1991a) A novel protein kinase homolog essential for protein sorting to the yeast lysosome-like vacuole. Cell 64:425-437

Herman PK, Stack JH, Emr SD (1991b) A genetic and structural analysis of the yeast Vps15 protein kinase: evidence for a direct role of Vps15p in vacuolar protein delivery. EMBO J 10:40494060

Howden R, Park SK, Moore JM, Orme J, Grossniklaus U, Twell D (1998) Selection of T-DNA-tagged male and female gametophytic mutants by segregation distortion in Arabidopsis. Genetics 149:621-631

Ischebeck T, Stenzel I, Heilmann I (2008) Type B phosphatidylinositol-4-phosphate 5-kinases mediate Arabidopsis and Nicotiana tabacum pollen tube growth by regulating apical pectin secretion. Plant Cell 20:3312-3330

Johnson MA, Preuss D (2002) Plotting a course: multiple signals guide pollen tubes to their targets. Dev Cell 2:273-281

Kihara A, Noda T, Ishihara N, Ohsumi Y (2001) Two distinct Vps34 phosphatidylinositol 3-kinase complexes function in autophagy and carboxypeptidase Y sorting in Saccharomyces cerevisiae. J Cell Biol 152:519-530

Kim DH, Eu YJ, Yoo CM, Kim YW, Pih KT, Jin JB, Kim SJ, Stenmark H, Hwang I (2001) Trafficking of phosphatidylinositol 3-phosphate from the trans-Golgi network to the lumen of the central vacuole in plant cells. Plant Cell 13:287-301

Lee Y, Bak G, Choi Y, Chuang WI, Cho HT (2008a) Roles of phosphatidylinositol 3-kinase in root hair growth. Plant Physiol 147:624-635

Lee Y, Kim ES, Choi Y, Hwang I, Staiger CJ, Chung YY, Lee Y (2008b) The Arabidopsis phosphatidylinositol 3-kinase is important for pollen development. Plant Physiol 147:1886-1897

Leshem Y, Seri L, Levine A (2007) Induction of phosphatidylinositol 3-kinase-mediated endocytosis by salt stress leads to intracellular production of reactive oxygen species and salt tolerance. Plant $\mathbf{J}$ 51:185-197

Li XG, Su YH, Zhao XY, Li W, Gao XQ, Zhang XS (2010) Cytokinin overproduction-caused alteration of flower development is partially mediated by CUC2 and CUC3 in Arabidopsis. Gene 450:109-120

McCormick S (2004) Control of male gametophyte development. Plant Cell 16(Suppl):142-153

Monteiro D, Castanho Coelho P, Rodrigues C, Camacho L, Quader H, Malho R (2005) Modulation of endocytosis in pollen tube growth by phosphoinositides and phospholipids. Protoplasma 226:31-38

Mueller-Roeber B, Pical C (2002) Inositol phospholipid metabolism in Arabidopsis. Characterized and putative isoforms of inositol phospholipid kinase and phosphoinositide-specific phospholipase C. Plant Physiol 130:22-46

Panaretou C, Domin J, Cockcroft S, Waterfield MD (1997) Characterization of $\mathrm{p} 150$, an adaptor protein for the human phosphatidylinositol (PtdIns) 3-kinase. Substrate presentation by phosphatidylinositol transfer protein to the p150. Ptdins 3-kinase complex. J Biol Chem 272:2477-2485

Petiot A, Faure J, Stenmark H, Gruenberg J (2003) PI3P signaling regulates receptor sorting but not transport in the endosomal pathway. J Cell Biol 162:971-979 
Qin G, Ma Z, Zhang L, Xing S, Hou X, Deng J, Liu J, Chen Z, Qu LJ, $\mathrm{Gu} \mathrm{H}$ (2007) Arabidopsis AtBECLIN 1/AtAtg6/AtVps30 is essential for pollen germination and plant development. Cell Res 17:249-263

Roth MG (2004) Phosphoinositides in constitutive membrane traffic. Physiol Rev 84:699-730

Samaj J, Muller J, Beck M, Bohm N, Menzel D (2006) Vesicular trafficking, cytoskeleton and signalling in root hairs and pollen tubes. Trends Plant Sci 11:594-600

Sbrissa D, Shisheva A (2005) Acquisition of unprecedented phosphatidylinositol 3, 5-bisphosphate rise in hyperosmotically stressed 3T3-L1 adipocytes, mediated by ArPIKfyve-PIKfyve pathway. J Biol Chem 280:7883-7889

Sousa E, Kost B, Malho R (2008) Arabidopsis phosphatidylinositol-4monophosphate 5-kinase 4 regulates pollen tube growth and polarity by modulating membrane recycling. Plant Cell 20: 3050-3064

Stack JH, Herman PK, Schu PV, Emr SD (1993) A membraneassociated complex containing the Vps 15 protein kinase and the Vps34 PI 3-kinase is essential for protein sorting to the yeast lysosome-like vacuole. EMBO J 12:2195-2204

Tian GW, Chen MH, Zaltsman A, Citovsky V (2006) Pollen-specific pectin methylesterase involved in pollen tube growth. Dev Biol 294:83-91

Wang XL, Xu YH, Peng CC, Fan RC, Gao XQ (2009) Ubiquitous distribution and different subcellular localization of sorbitol dehydrogenase in fruit and leaf of apple. J Exp Bot 60:1025-1034
Weisman LS (2003) Yeast vacuole inheritance and dynamics. Annu Rev Genet 37:435-460

Welters P, Takegawa K, Emr SD, Chrispeels MJ (1994) AtVPS34, a phosphatidylinositol 3-kinase of Arabidopsis thaliana, is an essential protein with homology to a calcium-dependent lipid binding domain. Proc Natl Acad Sci USA 91:11398-11402

Whitley P, Hinz S, Doughty J (2009) Arabidopsis FAB1/PIKfyve proteins are essential for development of viable pollen. Plant Physiol 151:1812-1822

Xue HW, Chen X, Mei Y (2009) Function and regulation of phospholipid signalling in plants. Biochem J 421:145-156

Yan Y, Backer JM (2007) Regulation of class III (Vps34) PI3Ks. Biochem Soc Trans 35:239-241

Yorimitsu T, Klionsky DJ (2005) Autophagy: molecular machinery for self-eating. Cell Death Differ 12:1542-1552

Zhang Y, McCormick S (2007) A distinct mechanism regulating a pollen-specific guanine nucleotide exchange factor for the small GTPase Rop in Arabidopsis thaliana. Proc Natl Acad Sci USA 104:18830-18835

Zhang Y, McCormick S (2010) The regulation of vesicle trafficking by small GTPases and phospholipids during pollen tube growth. Sex Plant Report 23:87-93

Zonia L, Munnik T (2008) Vesicle trafficking dynamics and visualization of zones of exocytosis and endocytosis in tobacco pollen tubes. J Exp Bot 59:861-873 